\title{
Air Shower Measurements with LOFAR
}

\author{
A. Horneffer ${ }^{*, a}$, L. Bähren ${ }^{\text {a }}$, S. Buitink ${ }^{\mathrm{a}}$, H. Falcke ${ }^{\mathrm{a}, \mathrm{b}}$, J.R. Hörandel $^{\mathrm{a}}$, J. Kuijpers ${ }^{\mathrm{a}}$, S. Lafebre ${ }^{\mathrm{a}}$, A. Nigla ${ }^{\mathrm{a}}$, \\ O. Scholten ${ }^{\mathrm{c}}$, K. Singh ${ }^{\mathrm{a}, \mathrm{c}}$ \\ ${ }^{a}$ Department of Astrophysics/IMAPP, Radboud University Nijmegen, 6500 GL Nijmegen, The Netherlands \\ ${ }^{b}$ ASTRON, 7990 AA Dwingeloo, The Netherlands \\ ${ }^{c}$ Kernfysisch Versneller Instituut, NL-9747 AA Groningen, The Netherlands
}

\begin{abstract}
Air showers from cosmic rays emit short, intense radio pulses. LOFAR is a new radio telescope, that is being built in the Netherlands and Europe. Designed primarily as a radio interferometer, the core of LOFAR will have a high density of radio antennas, which will be extremely well calibrated. This makes LOFAR a unique tool for the study of the radio properties of single air showers.

Triggering on the radio emission from air showers means detecting a short radio pulse and discriminating real events from radio interference. At LOFAR we plan to search for pulses in the digital data stream - either from single antennas or from already beam-formed data - and calculate several parameters characterizing the pulse shape to pick out real events in a second stage. In addition, we will have a small scintillator array to test and confirm the performance of the radio only trigger.
\end{abstract}

Key words:

LOFAR, Cosmic Rays

\section{Introduction}

It has been known since 1965 that cosmic ray air showers emit short radio pulses 1]. LOPES, a LOFAR Prototype Station, was the first experiment that has proven that with fast ADCs and modern computer technology it is possible to measure these radio pulses even in the presence of relatively strong RFI 12 .

LOFAR, the Low Frequency Array, is a new radio telescope for the frequency range of $10 \mathrm{MHz}$ to $270 \mathrm{MHz}$, that is being built in the Netherlands and Europe. Designed as a radio interferometer it will consist of more than 40 stations with fields of comparatively simple antennas. These stations are placed densely in the core of LOFAR and at increasing distances the further away from the core a station is. The core will have at least 18 stations of 48/96 dual/single polarization antennas in a roughly $2 \mathrm{~km}$ by $3 \mathrm{~km}$ area. To enable the radio astronomy application LOFAR will have a very

\footnotetext{
* Corresponding author.

Email address: A.Horneffer@astro.ru.nl (A. Horneffer)

${ }^{1}$ radio frequency interference

Preprint submitted to ARENA Proceedings
}

precise calibration. While this setup would not be cost-effective for pure air shower measurements it gives us an unique possibility to study the radio emission from single air showers in great detail. Figure 1 shows the different energy ranges in which LOFAR will measure cosmic rays. The HECR 2 and the VHECR 3 mode both measure air showers, they differ only in the method of triggering, but share the same post-processing and analysis software.

\section{LOFAR Air Shower Trigger}

Air shower radio pulses are short $(<20 \mathrm{~ns})$ pulses, that are beamed in the propagation direction of the air shower and illuminate an area comparable to the size of the particle disc. In addition the radio pulses do not arrive on the ground in a plane but with some curvature. Compared to a plane wave the radio pulses arrive later with increasing distance from the shower axis. These properties can be used to distinguish air shower pulses from pulsed RFI.

\footnotetext{
${ }^{2}$ high energy cosmic rays

${ }^{3}$ very high energy cosmic rays
} 


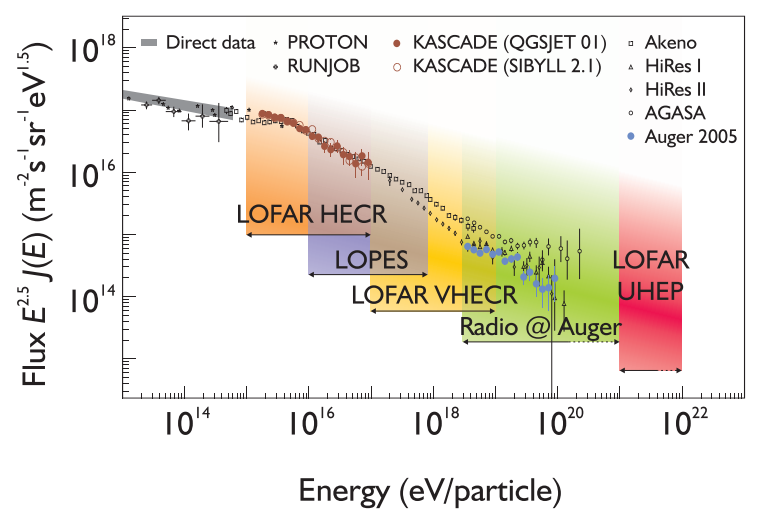

Figure 1: Cosmic ray energy spectrum with the measurement modes of LOFAR. In the HECR mode we look for air showers in beam-formed data, in VHECR mode we look for air showers in single channel data and in UHEP mode we look for radio pulses from particles hitting the moon. LOPES and Auger are related experiments.

In LOFAR there are to paths for the data. The signals from each dipole are digitized and then are both sent to the so called transient buffer boards (TBB) for storage in a memory ring-buffer and are processed in a continuous data stream. In this stream the data is converted into frequency bands, these bands are then beam-formed in a given direction at the station level and the beam-formed data from each station is sent to a central processing facility (CEP).

In the HECR mode we look at the beam-formed data from single stations. At CEP the beam-formed data is converted back into time-domain for full time resolution and searched for air shower candidate pulses. When a suitable candidate is found the data from the corresponding TBBs is saved to a file for off-line processing. As the beam-forming reduces the solid angle but increases the sensitivity, this mode is useful for low energy showers.

At higher energies, in the VHECR mode, we look for pulses in the data streams of single channels. This is done in three levels: the first level runs on the hardware of the TBBs. There the data from each channel is first filtered with up to three so called infinite impulse response (IIR) filters, that can be configured as high-pass, low-pass, or notch filters. The filtered data is then searched for pulses by evaluating the following equation:

$$
\left|x_{i}\right|>\mu_{i}+a \sigma_{i}
$$

(With $x_{i}$ the input data, $\mu_{i}$ and $\sigma_{i}$ the mean and standard deviation of $\left|x_{i}\right|$, and $a$ a parameter.) As the input data is Gaussian distributed $\mu_{i}$ is proportional to $\sigma_{i}$, so this equation can be simplified to:

$$
\left|x_{i}\right|>b \mu_{i}
$$

which greatly eases implementation on the TBBs. For every $x_{i}$ above threshold a counter is increased by 4 and decreased by one otherwise, unless it is zero. So by testing if this counter is above a given value one can detect pulses in which several values close to each other are above threshold. If such a pulse is detected the pulse parameters position in time, height, width, sum, average before and average after the pulse are calculated and sent in a message to the computer controlling the station. On this computer the second trigger level runs. In this an incoming message is first checked if the pulse parameters disqualify a pulse for an air shower candidate. With the remaining pulses a simple coincidence check is done to detect air shower candidates. If the available computing power permits we then can do a direction fit or look at the pulse height pattern on the ground to identify RFI pulses. If a good air shower candidate is identified all TBBs of the station are notified and their data are written to disk. Information about weak and good candidates is sent to CEP, which then can decide if a coincidence of weak pulses in several stations indicates an air shower in between them, or if a strong air shower warrants saving the data from additional stations.

\section{Test Measurements}

Since late 2007 there is already a test setup available, with one station of 48 dual polarization antennas and three stations with 16 antennas each. Two stations are equipped with TBBs that can collect data from 16 antennas each, for a total of 64 channels. Figure 2 shows a dynamic spectrum generated from LOFAR time-series data. Except for the short-wave band and one FM transmitter there is little RFI background for most of the time. Only at a few time intervals there was strong RFI present, that would make air shower measurements impossible.

With IIR filters one can do frequency filtering of a data stream in the time-domain without a Fourier transform of the data. They work by evaluating:

$$
\begin{aligned}
x_{i}^{\prime}= & b_{0} x_{i}+b_{1} x_{i-1}+b_{2} x_{i-2} \ldots \\
& -a_{1} x_{i-1}^{\prime}-a_{2} x_{i-2}^{\prime}-a_{3} x_{i-3}^{\prime} \cdots
\end{aligned}
$$




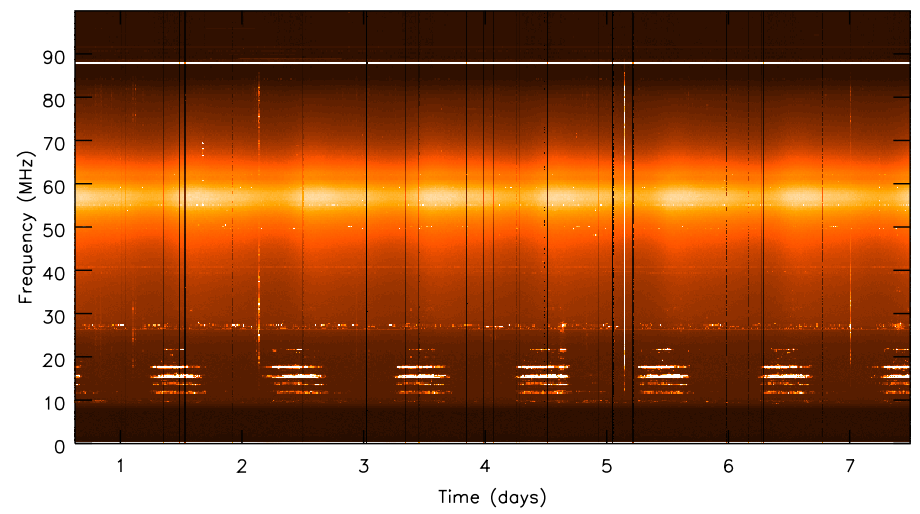

Figure 2: Dynamic spectrum generated from LOFAR time-series data. The white horizontal line at $88 \mathrm{MHz}$ is caused by a FM-transmitter, the white stripes between $8 \mathrm{MHz}$ and $20 \mathrm{MHz}$ by short-wave transmitters. Black vertical lines are caused by problems in the prototype data acquisition software, white vertical lines (e.g. at 5.1 days) show times of intense interference. The daily brightness variation is due to the galactic plane moving in and out of the field of view.
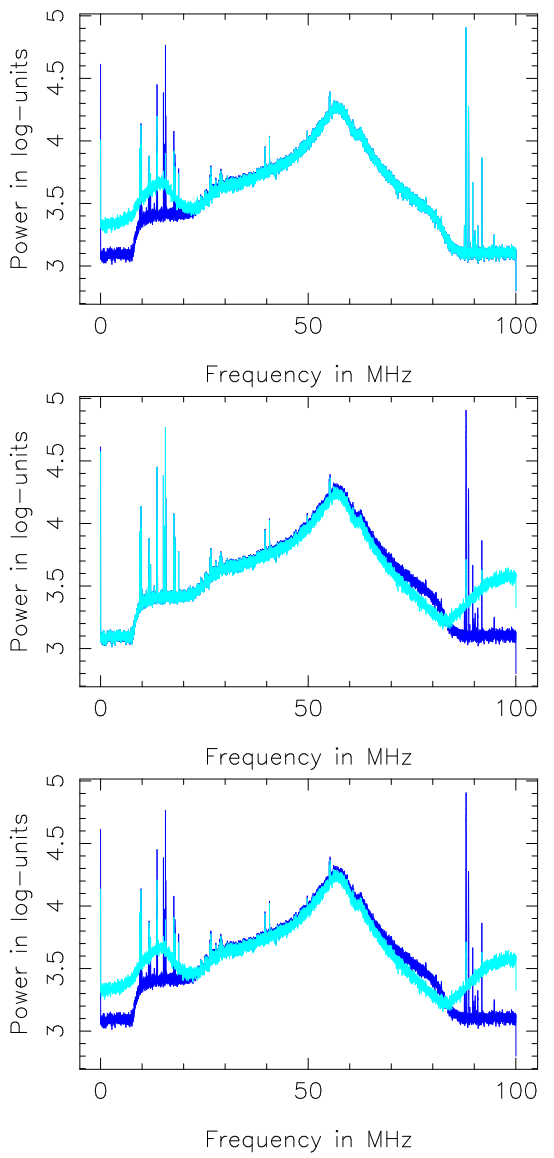

Figure 3: Effects of the digital IIR filters on the spectrum: unmodified spectrum in dark color and in light color: top: spectrum with notch-filter at $15 \mathrm{MHz}$; middle: spectrum with notch-filter at $88 \mathrm{MHz}$; bottom: spectrum with both filters. where $x_{i}^{\prime}$ represents the filtered, output data and $x_{i}$ is the input data. They are called infinite impulse response because in general they are able to produce an infinite nonzero output from an input with only one nonzero sample. In LOFAR we implemented a three stage filter in which the parameters $b_{0}, b_{1}, b_{2}, a_{1}$, and $a_{2}$ can be set. By choosing different values for the parameters one can implement a high-pass, low-pass or notch filter. One problem when choosing filter parameters is that setting a filter close to an edge of the frequency band produces aliasing effects, that can distribute power from one frequency to a previously clean frequency. Figure 3 shows the effect of two notch filters for the short-wave band and the FM transmitter on the frequency spectrum. The aliasing effects are clearly visible, but the total RFI power is still reduced by the filters. Test of the effect on the signal to noise for the detection of a standard pulse added to different noise data showed that the $88 \mathrm{MHz}$ filter increases the SNR at all times, while the $15 \mathrm{MHz}$ slightly decreases the SNR during times of low RFI from the short-wave band, but enhances the SNR when significant short-wave band RFI is present. While a reduced SNR is not desirable, the increased SNR during times of strong RFI evens out changes of the SNR with time of day, which makes also the $15 \mathrm{MHz}$ filter desirable.

\section{Particle Detector Array}

In addition to the radio only trigger we will set up a small particle detector array in the LOFAR core. 


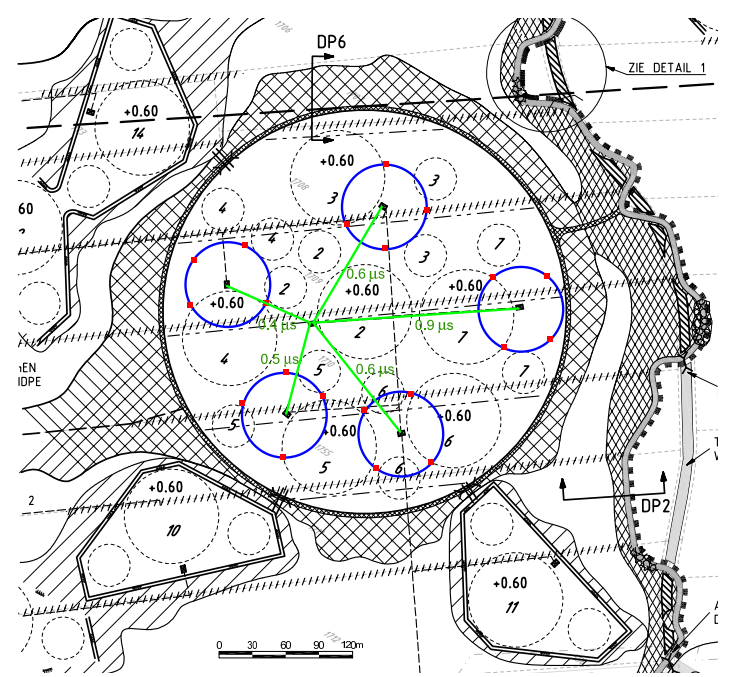

Figure 4: Layout of the LOFAR air shower array (LASA) in the LOFAR core. The small and big dashed circles show places of the LOFAR antenna fields. The five solid (blue) circles mark $50 \mathrm{~m}$ distance from the electronics stations and the (red) squares on these mark the planned positions for the particle detectors.

The purpose of this array is to test and confirm the performance of the radio trigger and provide additional information for the air shower analysis. Measuring with both, radio antennas and particle detectors will provide an important confirmation of our ability to select real air shower events only from radio data.

The array will consist of five stations with four detectors each, placed in the so called super-station. Figure 4 shows the layout of the array in the LOFAR center. The electronics of each station will be housed in electronic cabinets of the central LOFAR stations. Each station of four detectors will use the electronics developed for the HISPARC experiment [3] and have its own electronics with GPS-timing, trigger logic, and DAQ-PC. Thus it could be run as a stand-alone experiment, but it can also be triggered from outside, e.g. by the other stations. The data from all stations is collected at a central DAQ-PC, which generates combined eventfiles and can send a trigger signal to LOFAR.

The detectors are plastic scintillator detectors previously used in the KASCADE[4] experiment. Recent measurements revealed that the detectors do not emit significant RFI and the little emission they do emit is not expected to pose a problem for the regular operation of LOFAR.

\section{Summary}

The high sensitivity and excellent calibration of LOFAR will make it an unique tool for the measurements of radio emission from air showers. At LOFAR we will measure air showers in two modes: In the HECR mode we trigger on radio pulses in already beam-formed data, which will limit us to relatively low primary energies. In the VHECR mode we trigger on pulses in single channel data. This trigger algorithm will have three steps: on the hardware that writes the raw ADC data into a memory buffer, on the PC controlling a LOFAR station, and on the central processing facility. The algorithms for the first step and a preliminary version of the second step have already been implemented and the first tests have been successful. We also plan to set up a small particle detector array which will allow us to confirm that we can pick out real air shower events only with our radio trigger.

\section{References}

[1] J. V. Jelley et al., Nature 205 (1965) 327

[2] H. Falcke et al., LOPES collaboration, Nature 435 (2005) 313

[3] High School Project on Astro-Physics Research with Cosmics, http://www.hisparc.nl/

[4] T. Antoni et al., KASCADE collaboration, NIM A513 (2003) 490 